\title{
Currículo y Autonomía Pedagógica. Enseñanzas Mínimas, Comunes y Currículo Básico
}

\section{Curriculum and Pedagogical Autonomy. Minimum, Common Learning Requirements, and Core Curriculum}

\author{
Antonio Montero Alcaide * \\ Universidad de Sevilla, España
}

\section{DESCRIPTORES:}

Sistema educativo

Enseñanzas comunes

Enseñanzas mínimas

Currículo básico

Autonomía pedagógica

\section{RESUMEN:}

La naturaleza del currículo, considerado en el ámbito del sistema educativo, es objeto de atención desde distintos enfoques. Principalmente, con una caracterización formal o analítica del mismo, a partir de un conjunto de elementos propios y reunidos para dar forma a las enseñanzas mínimas o comunes que establecen el currículo básico. Con este punto de partida, el artículo adopta la forma de ensayo teórico para centrar el análisis en el diseño del currículo del sistema educativo español, considerada una panorámica desde la definición de las enseñanzas mínimas hasta la configuración de un currículo básico, con el trasfondo de la autonomía pedagógica. Como resultado principal, se constata, además de la imprecisión o desajuste de las enseñanzas mínimas, una limitación del currículo básico para responder a su genuina naturaleza: garantizar una formación común y la validez de las acreditaciones académicas correspondientes, en el marco de una autonomía pedagógica más declarada que factible. Por tanto, son objeto de discusión: la naturaleza de las enseñanzas que sostienen el currículo básico, la necesidad de una confluencia educativa que facilite acuerdos, al menos, sobre lo básico imprescindible, y la naturaleza condiciones y alcance del ejercicio de la autonomía pedagógica.
\end{abstract}

\section{KEYWORDS: \\ Educational system \\ Minimum learning requirements \\ Common learning requirements \\ Basic curriculum \\ Pedagogical autonomy}

\begin{abstract}
:
The essential nature of the curriculum within the sphere of the Educational System is studied from different perspectives. One of them is its formal or analytical conformation itself, gathering elements to outline the minimum or common learning requirements in order to determine the core curriculum. From here, the article is shaped as a theoretical essay focusing on the analysis of curriculum design in the Spanish Educational System; an overview from the definition of what minimum requirements really mean to the configuration of a core curriculum with the concept of pedagogical autonomy as a background to it all. As a result, it is established that the concept of minimum learning requirements is inaccurate and the core curriculum itself cannot ensure a common education and certification, besides the fact that pedagogical autonomy is more an aim than a reality. Therefore, the purpose is to study the true nature of the learning supporting the core curriculum, the need to reach some sort of agreements, at least about what is basic and essential, and the nature, conditions and limits of pedagogical autonomy.
\end{abstract}




\section{A modo de introducción: El currículo del sistema educativo como objeto de análisis}

La naturaleza, el alcance o el concepto de currículo, en el marco de distintas teorías, modelos y enfoques sobre el mismo, cuentan con una consolidada atención además de sustantivos y extensos antecedentes que afirman su entidad. En el sistema educativo español se advierte, entonces, una de las acepciones curriculares que Angulo (1994) propuso en momentos de diseño y desarrollo del currículo establecido en la Ley Orgánica de Ordenación General del Sistema Educativo (1990). Es el caso de una configuración, a partir de varios elementos característicos (competencias, objetivos, contenidos, métodos pedagógicos y didácticos, criterios y estándares de evaluación), cuyo sentido más propio es el de dar referencia a los procesos formales de enseñanza y de aprendizaje, desarrollados con distintos grados de autonomía pedagógica a partir de esa conformación curricular. Además de garantizar la educación común que conduce a la obtención de los reconocimientos y las titulaciones del sistema educativo.

La selección del conocimiento relevante, de la cultura social, que ha de conformar el currículo es una cuestión históricamente controvertida. No solo por la dificultad de los acuerdos o consensos en que debe sostenerse, sino por la propia indefinición, además de la amplitud, del conocimiento básico imprescindible (Coll, 2006) en la educación obligatoria. Es más, el currículo, en tanto que construcción social, lleva al análisis crítico (Penalva, 2007) de los principios que sustentan la apertura y flexibilidad curricular, así como de las consecuencias educativas para la formación común en la educación obligatoria. En este mismo sentido, el currículo crítico y participativo figura como una de las dimensiones para mejorar la democracia y la justicia social en los centros (Belavi y Murillo, 2020); toda vez que, sobre todo a partir de los métodos didácticos y de las prácticas docentes, con el marco de un currículo integrado para la democracia, pueden articularse procesos de construcción conjunta y compartida de tal currículo, tanto en su diseño como en su desarrollo. Considerado este último también como un proceso de construcción emergente del currículo.

Ha de subrayarse, por otra parte, el carácter educativo y social de la formación común (Gimeno, 2005), su doble naturaleza: educación básica terminal pero también preparatoria de estudios posteriores (Abiétar y Navas, 2017); así como el alcance no solo compensador, sino además transformador, del currículo en función del acceso del alumnado a la escolaridad en distintas condiciones y situaciones de partida. Si bien, tales intenciones, para tomar forma o progresivo desarrollo, requieren la elaboración más o menos pertinente del currículo, y, sobre todo y en mayor medida, precisan prácticas educativas que procuren respuestas adecuadas a las también distintas necesidades educativas del alumnado. Dado que no deben proclamarse efectos taumatúrgicos, casi prodigiosos, de las prescripciones normativas curriculares, tan cambiantes e inestables en nuestro sistema educativo, en el desempeño cotidiano de las prácticas docentes y en los procesos que conllevan cambios y mejora de las mismas. Principalmente, cuando entre los aspectos del desarrollo profesional docente no tienen una presencia significativa elementos sociales y culturales directamente relacionados con la educación obligatoria y, por ello, relevantes para la formación del profesorado (Martínez y Rodríguez, 2017).

El estudio y la investigación sobre el currículo, por otra parte, cuentan con un marco, principalmente en el ámbito anglosajón, desde las últimas décadas del siglo pasado, con una perspectiva histórica, la Historia del Currículum, vinculada a la Historia de la Educación. Tal historia subraya, ante las prerrogativas ciudadanas en la configuración de los estados, vínculos entre marcos constitucionales y curriculares (Tröhler, 2017) que son de interés por la adelantada entidad del currículo del sistema educativo. En el caso español, el análisis del mismo, desde la descentralización educativa, las competencias estatales y autonómicas para su definición, la política educativa y la normativa curricular de las Administraciones con las enseñanzas mínimas, comunes o básicas, han sido objeto asimismo de interés (Frías, 2007; González, 2004).

Expuestas estas consideraciones, con el presente trabajo, a modo de ensayo teórico, se opta por un objeto de análisis centrado en el diseño del currículo del sistema educativo español, a partir de una evolución del mismo desde la definición de las enseñanzas mínimas hasta la configuración de un currículo básico. La distribución de competencias en el diseño curricular permitirá, entonces, apreciar el grado de autonomía pedagógica resultante, a partir de las finalidades declaradas cuando tal currículo se establece formalmente en el sistema. Dos son, principalmente, tales propósitos: asegurar la formación común del alumnado y la validez de las acreditaciones y titulaciones a que conduce. Por lo que se abren o plantean 
cuestiones a fin de que el análisis y la discusión propicien conclusiones significativas: ¿responde el diseño del currículo, en el sistema educativo, a las intenciones con que se formula?, ¿qué aspectos del mismo facilitarían la adopción de consensos básicos y la colaboración administrativa?, ¿de qué modo la autonomía pedagógica converge con las finalidades del currículo básico y por qué adopta este un carácter prescriptivo?, ¿puede verificarse un cuestionamiento del carácter referencial del currículo?, ¿cabe disociar la autonomía pedagógica de las condiciones para su ejercicio?, ¿son necesarios procesos de revisión ante disfunciones en el uso de la autonomía o insuficiencias en los resultados tras la aplicación de la misma? A tales cuestiones se presta atención, por ello, en los apartados siguientes.

\section{Fundamentos del currículo básico en la ordenación del sistema educativo}

Con la publicación, en 1918, por Franklin Bobbit, del libro The Curriculum, el interés por lo que "debe enseñarse en la escuela" ha sido creciente y diversos los modos de entender la naturaleza y el alcance del currículo; con teorías y modelos, aplicados a su diseño y desarrollo, que suelen repartirse en tres grandes marcos: técnico, práctico y crítico, caracterizados de esta forma general, aunque caben distintos acercamientos en cada caso.

Como señala Tröhler (2017), una cuestión previa a la definición de las teorías sobre el currículo tiene que ver con el propio desarrollo de la Historia del Currículum y las conclusiones de las investigaciones internacionales y comparadas en educación. Algunos conceptos centrales y precursores del currículo, como los de ciudadano, nación y sociedad, que se abordaron desde marcos culturales norteamericanos, en las primeras décadas del siglo XX, toman distinta forma con la configuración de los estados-nación, por lo que pude plantearse que "los planes de estudio nacionales responden a un(a) idea(l) nacional diferente de la ciudadanía como portadores e implementadores de un orden social definido por preferencias culturales políticamente dominantes” (p. 203).

La configuración del currículo del sistema educativo participa, en buena medida, de los enfoques racionales o técnicos, con los conocidos antecedentes del desarrollo del currículo por objetivos, a partir de los trabajos de Bobbitt $(1918,1924)$, Tyler (1973) y de los desarrollos de Bloom $(1956,1964)$, Taba (1974) y Wheeler (1976). Aunque, claro está, cambien las perspectivas para la comprensión y el establecimiento de los objetivos de la educación.

La obra de R. W. Tyler (1949), Basic Principles of Curriculum and Instruction (con traducción española, Principios básicos del currículum, de 1973) establece cuatro cuestiones básicas a las que debe atender el desarrollo de cualquier currículo o "plan de enseñanza" (pp. 7-8):

- ¿Qué fines desea alcanzar la escuela?

- De todas las experiencias educativas que pueden brindarse, ¿cuáles ofrecen mayores probabilidades de alcanzar esos fines?

- ¿Cómo se pueden organizar de manera eficaz esas experiencias?

- ¿Cómo podemos comprobar si se han alcanzado los objetivos supuestos?

Por lo que resulta indudable la influencia de esta consideración racional o técnica del currículo en la definición del mismo en el sistema educativo.

Hilda Taba, en 1962, al publicar Curriculum development: Theory and practice (Elaboración del currículo, en la traducción española de 1974), cuestiona la naturaleza del currículo directamente vinculada a los objetivos y contenidos de la educación y afirma la necesidad de incorporar, asimismo, o de reforzar significativamente, los aspectos referidos al aprendizaje, de tal manera que no resulten ajenos "currículo" y "método":

Todos los currículos, no importa cuáles fuesen sus estructuras particulares, se componen de ciertos elementos. Generalmente se parte de una determinación de metas y objetivos especificos, lo que indica algún tipo de selección y organización del contenido, y destaca ciertos 
modelos de aprendizaje y enseñanza, sea por exigencia de los objetivos o de la organización del contenido. Finalmente, incluye un programa de evaluación de los resultados. (p. 24)

La autora adelanta y subraya a la vez el carácter sistémico del currículo, ya que cualquier decisión que se adopte sobre alguno de sus elementos no queda aislada, sino que afecta a los demás elementos comprendidos en un todo. En cualquier caso, Taba afirma que la elaboración del currículo requiere un "juicio ordenado" para la adopción de decisiones y propone un orden de pasos, en el que se advierten los elementos básicos adelantados por Tyler (1949, p. 24):

Paso 1: Diagnóstico de las necesidades.

Paso 2: Formulación de objetivos.

Paso 3: Selección de contenido.

Paso 4: Organización del contenido.

Paso 5: Selección de actividades de aprendizaje.

Paso 6: Organización de las actividades de aprendizaje.

Paso 7: Determinación de lo que se va a evaluary de las maneras y medios para hacerlo.

Finalmente, D. K. Wheeler se propone, con su trabajo Curriculum process (la traducción española, de 1976, se titula El desarrollo del currículum escolar), ofrecer un esquema lógico para considerar sistemáticamente los problemas del currículo, bastante coincidente con el de Tyler (1949). La inspiración conductista es manifiesta puesto que el autor sostiene que la educación tiene como fin modificar la conducta y entiende el currículo a partir de pretensiones -planificadas, deliberadas y sistemáticas- "de inculcar modelos de conducta aceptados (conocimientos, habilidades, hábitos, sensibilidad, actitudes y valores), y de inhibir los no aceptados" (p. 15). En tanto que el propio currículo se considera como "las experiencias planificadas que se ofrecen al alumno bajo la tutela de la escuela” (p.15). El esquema lógico adelantado por el autor se reparte, entonces, en cinco fases (p. 36):

Fase 1: Selección de metas, fines y objetivos.

Fase 2: Selección de experiencias que puedan contribuir a alcanzar esos fines, metas y objetivos.

Fase 3: Selección de contenidos (materias) a través de los cuales se ofrecen determinados tipos de experiencia.

Fase 4: Organización e integración de experiencias y contenidos en el proceso de enseñanza-aprendizaje dentro del aula y de la escuela.

Fase 5: Evaluación de la eficacia de todos los aspectos de las fases 2, 3 y 4 para alcanzar las metas detalladas en la fase 1.

Se reitera, por ello, la clara influencia de estas cuestiones, pasos o fases, referidos a la estructura del currículo, en la configuración que este adopta en los sistemas educativos.

La relación del currículo con problemas de acción (Clark y Yinger, 1980; Schön, 1983) aminora las determinaciones técnicas y formales ante el efecto mayor de las contingencias y las singularidades que sitúan los procesos educativos, así como de la naturaleza práctica de los procesos de diseño y desarrollo del currículo (Schwab, 1983; Stenhouse, 1987). Este último autor formula, así, una definición clásica del currículo desde las teorías prácticas: "Un currículum es una tentativa para comunicar los principios y rasgos esenciales de un propósito educativo, de forma tal que permanezca abierto a discusión crítica y pueda ser trasladado efectivamente a la práctica" (p. 29). De modo que la deliberación curricular se establece como fundamento de las prácticas, para hacer explícitos y argumentados los fines que guían los procesos y las acciones (identificar los objetivos, establecer patrones metodológicos, determinar los criterios y procedimientos de evaluación, adoptar formas de organización) concordantes con los principios curriculares. El conocimiento generado desde la reflexión sobre las prácticas -además de la incertidumbre, la complejidad y la singularidad de las situaciones educativas- necesita, por tanto, de un currículo abierto y flexible. Sin embargo, Stenhosue (1987) propone un esquema desde una premisa mínima: "Como mínimo, un 
currículum ha de proporcionar una base para planificar un curso, estudiarlo empíricamente y considerar los motivos de su justificación” (p. 30). Esta triple naturaleza es detallada del modo siguiente (p. 30):

A. En cuanto a proyecto:

- Principios para la selección de contenido: qué es lo que debe aprenderse y enseñarse.

- Principios para el desarrollo de una estrategia de enseñanza: cómo debe aprenderse y enseñarse.

- Principios acerca de la adopción de decisiones relativas a la secuencia.

- Principios a base de los cuales diagnosticar los puntos fuertes y débiles de los estudiantes individualmente considerados y diferenciar los principios generales 1, 2 y 3 antes señalados, a fin de ajustarse a los casos individuales.

B. En cuanto a estudio empírico:

- Principios a base de los cuales estudiar y evaluar el progreso de los estudiantes.

- Principios a base de los cuales estudiar y evaluar el progreso de los profesores.

- Orientación en cuanto a la posibilidad de llevar a cabo el currículum en diferentes situaciones escolares, contextos relativos a los alumnos, medios ambientes y situaciones de grupo entre los alumnos.

- Información sobre la variabilidad de efectos en diferentes contextos y sobre diversos alumnos y comprender las causas de la variación.

C. En relación a la justificación:

Una formulación de la intención o la finalidad del currículo que sea susceptible de examen crítico. Desde el marco de las teorías críticas, ideológicas y emancipadoras, Kemmis (1988) fundamenta una aproximación al currículo desde su carácter de construcción histórica y social. Además del cuestionamiento o la revisión de las funciones o del papel de los estados para determinar el sentido de la educación, así como de la utilidad del currículo y de la escolarización para servir a distintos intereses y valores. La función reproductora del currículo, pareja al desarrollo de escolarización, ocupó el interés de autores con fundamentos críticos precursores (Apple, 1981, 1982, 1986; Bordieu y Passeron, 1977; Giroux, 1981, 1983). Se consideran, por tanto, dos funciones principales de la educación: la reproducción de desenvolvimientos (conductas, interacciones) que responden a necesidades sociales predefinidas, y similar reproducción de conocimientos compartidos que den sustento a la vida social. Luego el currículo presta un servicio a propósito y las teorías críticas procuran dinámicas de cuestionamiento tanto en el ámbito de las ideas como en el de las prácticas; dada una fuerte determinación ideológica: "El currículum escolar, como otros aspectos de la vida social, está formado y moldeado ideológicamente. Así, las formas dominantes del currículum escolar reflejan las formas ideológicas dominantes en la sociedad” (Kemmis, 1988, p. 22).

En este sentido, Tröhler (2017), ocupado en la relación entre las constituciones nacionales y las leyes educativas que establecen los currículos, sostiene que las élites políticas, para establecer un nuevo orden social, han de estimar elementos preexistentes, y cita a Zimmer (2003) para señalar que tales élites debieron "construir sus ideologías de modo que llegaran al público, lo cual depende en parte de su capacidad de conectar, de forma significativa, con esquemas culturales y morales preexistentes" (p. 5).

Penalva (2007) aborda el marco crítico del currículo desde una orientación sociológica principalmente centrada en la explicación de "los procesos por los que los grupos sociales que participan en la educación seleccionan los conocimientos y las creencias, y cómo sus discursos pasan a formar parte de la escuela y, en último término, de la ideología social dominante" (p. 4). Luego concierne a los estudios sobre el currículo indagar sobre los procesos de construcción del conocimiento, considerada la escuela en el ámbito de las instituciones sociales que pueden legitimar, precisamente por la configuración de los contenidos curriculares y la evaluación de los mismos, culturas hegemónicas. 
Al cabo, la política educativa no es ajena, sino derivada, de la política general del Estado y, por tanto, sobre el currículo escolar ejercen influencia destacada, como señala Frías (2007), las orientaciones y los enfoques políticos. Cuestión, por otra parte, bien apreciable en el caso del sistema educativo español, que afecta a la falta de estabilidad y a la ausencia de consenso sobre cuestiones curriculares.

El currículo básico del sistema educativo toma referencias, si bien en diferente grado, de los marcos teóricos adelantados, pero es asimismo pertinente un acercamiento desde las que Angulo (1994), consideró "acepciones" del currículo, para entenderlo en un continuo de la "representación" a la "acción". Por ello, propone tres consideraciones del currículo: una cercana al "contenido", otra próxima a la "planificación educativa" y la tercera como "realidad interactiva". Útiles, las tres, para entender y fundamentar específicamente el currículo del sistema educativo.

El carácter del currículo como contenido se materializa en distintas formas, generalmente próximas a las materias de aprendizaje (Gagné, 1968) o al curso de estudios (Taylor y Richards, 1985). Asimismo, su consideración como resultados de aprendizaje para prescribir la instrucción (Johnson, 1967) es de especial interés por la incorporación de estándares y resultados de aprendizaje evaluables a los elementos del currículo establecido, en el sistema educativo español, por la Ley Orgánica para la Mejora de la Calidad Educativa (2013). Angulo, en este sentido, utilizó la asimilación del currículo como contenido a los resultados de aprendizaje para caracterizar el propio currículo regulado en la Ley Orgánica de Ordenación General del Sistema Educativo (1990). Toda vez que el concepto normativo de currículo tiende a equiparar el currículo con indicadores de contenido cultural que se expresan para ser adquiridos mediante el aprendizaje del alumnado.

Por su parte, el currículo como planificación educativa sostiene una estructura del mismo, habitualmente considerada en la ordenación de los sistemas educativos, con elementos característicos. Es el caso de los objetivos de la enseñanza, de los contenidos a los que se aplica, de las orientaciones didácticas y de los criterios, indicadores o estándares de evaluación. Aunque, en este caso, importe no poco diferenciar entre el currículo planificado y el currículo en uso:

La planificación curricular no es, por ello, el currículum operativo, no son las acciones y las decisiones, pero las determina y vehicula. El currículum operativo o en uso es, pues, el resultado de llevar a la práctica las prescripciones del currículum planificado. (Angulo, 1994, p. 25)

En tanto que el currículo entendido como interacción no se vincula directamente con las prescripciones curriculares, sino que incluso puede mejorarlas a partir de la reinterpretación, recreación o desarrollo de aquellas en las prácticas educativas en el aula. Perspectiva, como las anteriores, que se considera en la construcción de teorías y modelos sobre el currículo.

De parecido modo, Gimeno (1989), en momentos de elaboración del currículo de la Ley Orgánica de Ordenación General del Sistema Educativo (1990), identificó cinco aspectos para el análisis del currículo. Cabe considerarlo, entonces, como una función social, toda vez que propicia vínculos entre la sociedad y la escuela. Más consabida y extendida es su naturaleza como proyecto o plan educativo, ya ideal o real, a partir de elementos característicos del mismo que traen causa de las primeras configuraciones del currículo (objetivos, contenidos, métodos pedagógicos y actividades didácticas, evaluación). También puede el currículo aplicarse a la expresión material del mismo -no solo como proyecto-, aproximándose a un carácter práctico que engarza con los procesos de enseñanza en el desarrollo del currículo. Y queda, además, una consideración del currículo como referencia discursiva, en ámbitos académicos o de investigación, asociado al alcance mayor de la educación o de ámbitos específicos de la misma.

El diseño del currículo, por otra parte, incumbe a la política educativa con razones que Gimeno Sacristán y Pérez Gómez (1999) vinculan a una cultura de la escuela ordenada a lo largo de la escolarización: El que desde la política educativa y desde la Administración, como instrumento de la misma, se intervenga en el diseño general del currículum es una necesidad derivada de la importancia de ordenar la cultura de la escuela a lo largo de un periodo prolongado de escolarización a través del que distribuir el contenido, enlazando adecuadamente sus tramos. (p. 266)

Sin embargo, distintas pueden ser las formas de elaborarlo y, sobre todo, la determinación que tal currículo conlleve. Reiterada es así la dialéctica entre el grado de apertura del currículo, la autonomía pedagógica de los centros para desarrollarlo o concretarlo en función de las distintas situaciones o necesidades 
educativas del alumnado, o el grado en que se lleva a término una regulación que asegure la aplicación del currículo básico. Gimeno (1989), tras sostener que el contenido es la condición lógica de la enseñanza, subraya el carácter de currículo como "selección cultural estructurada bajo claves psicopedagógicas de esa cultura que se ofrece como proyecto para la institución escolar” (p. 20).

Luego la cultura de la escuela, por la generalización de una educación básica graduada, la necesidad de establecer, organizar y controlar la selección de objetivos y contenidos educativos, las referencias y criterios para la evaluación del aprendizaje y de los logros educativos del alumnado, o la adecuación de las prácticas docentes tienen directa relación con los procesos de configuración del currículo.

De ahí que César Coll, poco antes de la definición del currículo de las enseñanzas de la Ley Orgánica de Ordenación General del Sistema Educativo (1990), considerase que el currículo define una orientación general de tal sistema, a partir de principios y fuentes de distintas naturalezas (ideológica, psicopedagógica, sociológica). De modo que el propio currículo las convierte en prescripciones educativas a fin de disponer de un instrumento que resulte útil y eficaz para las prácticas docentes:

El currículum es un eslabón que se sitúa entre la declaración de principios generales y su traducción operacional, entre la teoría educativa y la práctica pedagógica, entre la planificación y la acción, entre lo que se prescribe y lo que realmente sucede en las aulas. (Coll, 1987, p. 21)

Se dispone, por tanto, de marcos, enfoques y perspectivas relevantes que fundamentan el estudio y análisis del currículo básico del sistema educativo. Este, como habrá podido comprobarse, resulta más próximo a los modelos racionales o técnicos, a partir de una configuración analítica que reúne elementos característicos del currículo. La autonomía pedagógica toma como referencia las enseñanzas mínimas o básicas que, para las distintas áreas o materias, se establecen con la consideración de tales elementos curriculares. Se subraya, en definitiva, el acercamiento del currículo a los contenidos de la enseñanza, acompañados de otros aspectos, y a la planificación educativa resultante. Como consecuencia, la autonomía se aproxima a distintos niveles de concreción curricular o al desarrollo de competencias distribuidas y específicas sobre tal currículo, con menor relevancia de las teorías o enfoques que subrayan los problemas de acción, el currículo emergente desde las prácticas de enseñanza, o los acercamientos críticos de construcción y reconstrucción social del currículo. Estas últimas perspectivas, por otra parte, propiciarían una autonomía menos analítica con una configuración del currículo básico que estableciera referencias básicas poco cuestionadas por imprescindibles.

\section{La configuración del currículo en el sistema educativo español como resultado}

La distribución de competencias y funciones para la determinación del currículo es un aspecto de especial interés en este punto, dado que explica la configuración de las enseñanzas mínimas, de las enseñanzas comunes y del currículo básico.

Como punto de partida para la definición del currículo del sistema educativo español, ha de considerarse que, en la modernización de este, llevada a cabo por la Ley General de Educación y Financiamiento de la Reforma Educativa (1970), se estableció que correspondía al Gobierno, en materia de educación, "La reglamentación de todas las enseñanzas y la concesión o reconocimiento de los títulos correspondientes" (artículo 4.6). Por tanto, las competencias para establecer el currículo de las enseñanzas quedaban reservadas al Gobierno, con el doble objeto de definir una formación común y reconocer los títulos a que conducen las enseñanzas.

La Constitución española de 1978, en su artículo 27.5, vincula la garantía del derecho a la educación con una programación general de la enseñanza en la que se verifique la participación: "Los poderes públicos garantizan el derecho de todos a la educación, mediante una programación general de la enseñanza, con participación efectiva de todos los sectores afectados y la creación de centros docentes”. Asimismo, determina que el Estado tiene competencia exclusiva sobre la regulación de las condiciones de obtención, expedición y homologación de títulos académicos y profesionales y normas básicas para el desarrollo 
del artículo 27 de la Constitución, a fin de garantizar el cumplimiento de las obligaciones de los poderes públicos en esta materia (Artículo 149.1.30 a).

Por tanto, una cuestión sustantiva debe adelantarse ya: la configuración del currículo en el sistema educativo adquiere sentido, entre otros aspectos, por la acreditación de las titulaciones académicas y profesionales a que conducen las distintas enseñanzas. De ahí la necesidad de una formación común y la competencia exclusiva del Estado para establecer tanto las condiciones de obtención de los distintos títulos como la expedición y homologación de los mismos. No se desplaza, con ello, la consideración del currículo como producto cultural, como conocimiento socialmente organizado, sino que esta naturaleza, si se quiere hasta la identidad del currículo, toma forma y confluye en la definición de los elementos del mismo. Principalmente, en los contenidos y en la evaluación de los aprendizajes del alumnado, que han de ser comunes porque conducen a titulaciones académicas y profesionales. Todavía más, la configuración básica del currículo sostiene el derecho a la educación, por determinar la adquisición de esta mediante el patrón curricular de las distintas enseñanzas del sistema educativo.

Con estos precedentes generales, la primera referencia a las "enseñanzas mínimas" se hace con la promulgación, en 1980, de la Ley Orgánica por la que se regula el Estatuto de Centros Escolares. En su disposición adicional 2.b, se establece que, en todo caso, y por su propia naturaleza, corresponde al Estado: "La fijación de las enseñanzas mínimas y la regulación de las demás condiciones para la obtención, expedición y homologación de títulos académicos y profesionales válidos en todo el territorio español”. La vigencia de esta ley, afectada por un recurso de inconstitucionalidad, fue limitada y cinco años después, en 1985, la Ley Orgánica del Derecho a la Educación reproduce literalmente, en su disposición adicional 1.2.c), la disposición anterior, de 1980.

La inestabilidad del sistema educativo español, debida a la falta de consenso en la alternancia de los Gobiernos, hace que las enseñanzas mínimas registren los siguientes cambios en el tiempo, a partir de las distintas "leyes curriculares" que se han sucedido:

- Con la promulgación de la Ley de Ordenación General del Sistema Educativo (LOGSE, 1990), los aspectos básicos del currículo, tal como se refiere en su artículo 4, constituyen las enseñanzas mínimas.

- A partir del año 2002, publicada la Ley Orgánica de Calidad de la Educación (LOCE), se adopta el concepto de "enseñanzas comunes", en su artículo 8, y estas constituyen los aspectos básicos del currículo.

- La Ley Orgánica de Educación (LOE, 2006), recupera, en el artículo 6, la denominación de "enseñanzas mínimas” en similares términos a los de la Ley de Ordenación General del Sistema Educativo (1990).

- En 2013, promulgada la Ley Orgánica para la Mejora de la Calidad Educativa (LOMCE), su artículo único 4 modifica el artículo 6 de la Ley Orgánica de Educación (2006) y, como competencia del Gobierno, en su artículo único 5, por el que se añade un nuevo artículo 6 bis a la Ley Orgánica de Educación, alude al diseño del "currículo básico".

- Esta misma Ley Orgánica para la Mejora de la Calidad Educativa (2013), con su disposición final 2.7, deroga la disposición adicional 1.2.c) de la Ley Orgánica del Derecho a la Educación (LODE, 1985), que aludía directamente a las enseñanzas mínimas.

- Finalmente, en 2020, la Ley Orgánica por la que se modifica la Ley Orgánica 2/2006, de 3 de mayo, de Educación (LOMLOE), en su artículo único 4, modifica la redacción del artículo 6 de la Ley Orgánica de Educación (2006), dada por la Ley Orgánica para la Mejora de la Calidad Educativa (2013), y recupera la configuración del currículo a partir de las enseñanzas mínimas.

El desarrollo de tales configuraciones del currículo, en la panorámica del desarrollo normativo del mismo, se detalla en el cuadro siguiente y algunos aspectos del análisis resultan significativos. 
Cuadro 1

Caracterización del currículo en el sistema educativo español

LOGSE (1990)

Artículo 4

Se entiende por currículo el conjunto de objetivos, contenidos, métodos pedagógicos y criterios de evaluación de cada uno de los niveles, etapas, ciclos, grados y modalidades del sistema educativo que regulan la práctica docente.

El Gobierno fijará, en relación con los objetivos, expresados en términos de capacidades, contenidos y criterios de evaluación del currículo, los aspectos básicos de éste que constituirán las enseñanzas mínimas, con el fin de garantizar una formación común de todos los alumnos y la validez de los títulos correspondientes.

Los contenidos básicos de las enseñanzas mínimas, en ningún caso requerirán más del 55 por ciento de los horarios escolares para las Comunidades Autónomas que tengan lengua oficial distinta del castellano, y del 65 por ciento para aquéllas que no la tengan.

Las Administraciones educativas competentes establecerán el currículo de los distintos niveles, etapas, ciclos, grados y modalidades del sistema educativo, del que formarán parte, en todo caso, las enseñanzas mínimas.

\section{LOCE (2002)}

Artículo 8

Se entiende por currículo el conjunto de objetivos, contenidos, métodos pedagógicos y criterios de evaluación de cada uno de los niveles, etapas, ciclos, grados y modalidades del sistema educativo.

En relación con los objetivos, contenidos y criterios de evaluación del currículo, el Gobierno fijará las enseñanzas comunes, que constituyen los elementos básicos del currículo, con el fin de garantizar una formación común a todos los alumnos y la validez de los títulos correspondientes.
LOE (2006)

Artículo 6

Se entiende por currículo el conjunto de objetivos, competencias básicas, contenidos, métodos pedagógicos y criterios de evaluación de cada una de las enseñanzas.

Con el fin de asegurar una formación común y garantizar la validez de los títulos correspondientes, el Gobierno fijará, en relación con los objetivos, competencias básicas, contenidos y criterios de evaluación, los aspectos básicos del currículo que constituyen las enseñanzas mínimas.
A los contenidos de las enseñanzas comunes les corresponde en todo caso el 55 por 100 de los horarios escolares en las Comunidades Autónomas que tengan, junto con la castellana, otra lengua propia cooficial y el 65 por 100 en el caso de aquellas que no la tengan.

Las Administraciones educativas competentes establecerán el currículo de los distintos niveles, etapas, ciclos, grados y modalidades del sistema educativo, que deberá incluir las enseñanzas comunes en sus propios términos.
Los contenidos básicos de las enseñanzas mínimas requerirán el 55 por ciento de los horarios escolares para las Comunidades Autónomas que tengan lengua cooficial y el 65 por ciento para aquéllas que no la tengan.

Las Administraciones educativas establecerán el currículo de las distintas enseñanzas, del que formarán parte los aspectos básicos señalados anteriormente. Los centros docentes desarrollarán y completarán, en su caso, el currículo de las diferentes etapas y ciclos en uso de su autonomía.

Los títulos correspondientes a las enseñanzas serán homologados por el Estado y expedidos por las Administraciones educativas en las condiciones previstas en la ordenación normativa
Los títulos académicos y profesionales serán homologados por el Estado y expedidos por las Administraciones educativas en tas condiciones previstas por la ordenación normativa.
Los títulos académicos y profesionales serán homologados por el Estado y expedidos por las Administraciones educativas en las condiciones establecidas en la ordenación normativa.

\section{LOMCE (20013). Artículo único 4}

Se entiende por currículo la regulación de los elementos que determinan los procesos de enseñanza y aprendizaje para cada una de las enseñanzas.

El currículo estará integrado por los siguientes elementos:

- Los objetivos de cada enseñanza y etapa educativa.

- Las competencias, o capacidades para aplicar de forma integrada los contenidos propios de cada enseñanza y etapa educativa, con el fin de lograr la realización adecuada de actividades y la resolución eficaz de problemas complejos.

- Los contenidos, o conjuntos de conocimientos, habilidades, destrezas y actitudes que contribuyen al logro de los objetivos de cada enseñanza y etapa educativa y a la adquisición de competencias.

- Los contenidos se ordenan en asignaturas, que se clasifican en materias, ámbitos, áreas y módulos en función de las enseñanzas, las etapas educativas o los programas en que participen los alumnos y alumnas.

- La metodología didáctica, que comprende tanto la descripción de las prácticas docentes como la organización del trabajo de los docentes.

- Los estándares y resultados de aprendizaje evaluables.

- Los criterios de evaluación del grado de adquisición de las competencias y del logro de los objetivos de cada enseñanza y etapa educativa

Los títulos correspondientes a las enseñanzas serán homologados por el Estado y expedidos por las Administraciones educativas en las condiciones previstas en la ordenación normativa 


\section{LOMLOE (2020). Artículo único 4}

Se entiende por currículo el conjunto de objetivos, competencias, contenidos, métodos pedagógicos y criterios de evaluación de cada una de las enseñanzas.

Con el fin de asegurar una formación común y garantizar la validez de los títulos correspondientes, el Gobierno, previa consulta a las Comunidades Autónomas, fijará, en relación con los objetivos, competencias, contenidos y criterios de evaluación, los aspectos básicos del currículo, que constituyen las enseñanzas mínimas.

Las enseñanzas mínimas requerirán el 50 por ciento de los horarios escolares para las Comunidades Autónomas que tengan lengua cooficial y el 60 por ciento para aquellas que no la tengan.

Las Administraciones educativas establecerán el currículo de las distintas enseñanzas, del que formarán parte los aspectos básicos anteriormente señalados. Los centros docentes desarrollarán y completarán, en su caso, el currículo de las diferentes etapas y ciclos en el uso de su autonomía.

Las Administraciones educativas revisarán periódicamente los currículos para adecuarlos a los avances del conocimiento, así como a los cambios y nuevas exigencias de su ámbito local, de la sociedad española y del contexto europeo e internacional.

Los títulos correspondientes a las enseñanzas serán homologados por el Estado y expedidos por las Administraciones educativas en las condiciones previstas en la legislación vigente y en las normas básicas y específicas que al efecto se dicten.

Nota. Elaboración propia.

Es manifiesta la conformación del currículo del sistema educativo español, como ya se adelantó, desde modelos o enfoques racionales o técnicos, con los elementos fundamentales del mismo. El currículo, por tanto, se presenta como un conjunto de elementos cuya intención principal se ha hecho explícita: regular la práctica docente, en el caso de la Ley Orgánica de Ordenación General del Sistema Educativo (1990), o determinar los procesos de enseñanza y aprendizaje en el caso de la Ley Orgánica para la Mejora de la Calidad Educativa (2013). Tanto una como otra acción, regular las prácticas docentes o determinar los procesos de enseñanza y aprendizaje, atribuyen al currículo una intención prescriptiva cuyo efecto o cumplimiento cabe poner en cuestión. Ciertamente, las prescripciones curriculares pueden influir en las prácticas docentes -qué, para qué y cómo se enseña y se evalúa- e incluso regular su ejercicio, pero más consonantes y a propósito son otras finalidades igualmente explícitas: asegurar o garantizar una formación común, así como la validez de las titulaciones académicas y profesionales.

Las enseñanzas mínimas, presentes tanto en la Ley Orgánica de Ordenación General del Sistema Educativo (1990) como en la Ley Orgánica de Educación (2006) y en la Ley Orgánica de Modificación de la Ley Orgánica de Educación, se constituyen a partir de los aspectos básicos del currículo, fijados por el Gobierno en relación con los distintos componentes curriculares (objetivos, competencias, contenidos, métodos, criterios de evaluación). Mientras que las enseñanzas comunes, relacionadas con esos mimos componentes del currículo, son fijadas por el Gobierno y tales enseñanzas conforman los elementos básicos del currículo. Aunque pueda parecer un juego de palabras, tienen distinto alcance unos aspectos básicos del currículo, para constituir las enseñanzas mínimas, que unas enseñanzas comunes a fin de establecer los elementos básicos del currículo. El subrayado carácter común de las enseñanzas tiene bastante que ver con ello.

Así, cuando las Administraciones educativas competentes habían de establecer el currículo, en las distintas etapas, ciclos, grados o modalidades del sistema educativo, de tal currículo debían formar parte las enseñanzas mínimas; declaración más general que efectiva. Mientras que, en el caso de las enseñanzas comunes, ese currículo establecido por las distintas Administraciones educativas debía incluir las enseñanzas comunes "en sus propios términos".

Como cuestión de bastante relevancia, que afecta al diseño y desarrollo del currículo, han de considerarse los aspectos recogidos en el artículo 6 bis de la Ley Orgánica de Educación (2006), sobre "Currículo y distribución de competencias". Precisamente ese artículo 6 bis, incorporado por el artículo único.5 de la Ley Orgánica para la Mejora de la Calidad Educativa (2013), establece una detallada distribución de competencias sobre el currículo entre el Gobierno, las Administraciones educativas y los centros docentes, que sitúa las posibilidades de autonomía pedagógica de los centros. Si bien, las modificaciones introducidas 
por la Ley Orgánica de Modificación de la Ley Orgánica de Educación, reajustan tal distribución de competencias al establecer niveles de concreción del currículo vinculados a las enseñanzas mínimas.

Se ha constatado, por ello, una expresa evolución, en el sistema educativo español, desde los distintos niveles de concreción del currículo, a cargo de las Administraciones educativas y los centros - cuya virtualidad adelantó, en 1987, César Coll-, hasta la definición de nuevas competencias sobre el currículo que se establecen considerando dos referencias principales: el tipo de asignaturas y los elementos del currículo de las mismas. Es decir, un cambio sustantivo hizo pasar de los progresivos niveles de concreción del currículo a la distribución de distintas competencias sobre el mismo, en función del tipo de asignaturas establecidas por la Ley Orgánica para la Mejora de la Calidad de la Educación (2013) -troncales, específicas y de libre configuración autonómica- y de los elementos del currículo de las mismas -preferentemente, contenidos, criterios de evaluación y estándares de aprendizaje-. Como efecto, las competencias sobre el currículo del bloque de asignaturas troncales quedan atribuidas al Gobierno, así como sobre los criterios de evaluación y los estándares de las asignaturas específicas. Las Administraciones educativas adquieren competencias para determinar los contenidos de las asignaturas específicas, así como en el currículo de las asignaturas de libre configuración autonómica. Y los centros docentes pueden complementar los contenidos y diseñar e implantar métodos pedagógicos y didácticos propios. Tal es, por ello, el espacio de autonomía pedagógica resultante, principalmente centrada en el ámbito de las prácticas docentes, con distintas posibilidades (Assens et al. 2020), dado el carácter común del currículo y la determinación que, sobre el mismo, se ejerce por las Administraciones educativas en los elementos curriculares correspondientes a los contenidos y los criterios de evaluación de las enseñanzas. Este modelo de diseño curricular, ante una nueva modificación de la Ley Orgánica de Educación (2006), posterior a la introducida por la Ley Orgánica para la Mejora de la Calidad Educativa (2013), es objeto de revisión al desaparecer la organización de las áreas o materias en bloques de asignaturas y no constar los estándares de aprendizaje como elementos del currículo.

\section{Discusión, conclusiones y propuestas frente al escepticismo}

La ausencia de acuerdos mayores y estables (el consenso tal vez resulte inalcanzable) es un rasgo idiosincrásico del sistema educativo español. Y, por tanto, las propuestas que se sostienen en tal propósito pueden resultar afectadas por el escepticismo. Sin embargo, del mismo modo que decir lo obvio importa, la discusión sobre el estado de la cuestión y las conclusiones derivadas ayudan formular propuestas que confluyan en la configuración y mejora del currículo. Y esto, como sostiene Casanova (2012), "partiendo del principio básico de que cualquier mejora de la calidad educativa pasa por su traducción al diseño curricular que es lo que, efectivamente, llega a centros y aulas, es decir, a los estudiantes de cada época histórica” (p. 8).

Hacer viable la coherencia es un elemento prospectivo y no menor. En la revisión acometida de la identidad del currículo del sistema educativo, la finalidad básica del mismo es doble: garantizar una formación común de todo el alumnado y la validez de los títulos correspondiente. Este carácter garantista, que se mantiene constante en el vaivén de los cambios legislativos, obliga a considerar medidas o recursos que lo evidencien. La autonomía pedagógica, en tal sentido, no se opone a los propósitos principales del currículo, sino que propicia la consecución de estos mediante la adecuación de las respuestas educativas que materializan el currículo en los procesos de enseñanza y aprendizaje.

La definición de enseñanzas comunes puede estar guiada por el objeto de asegurar esa formación precisamente común, dado que, como sostiene Frías (2007), un currículo básico, formulado con excesiva generalización, puede desarrollarse de muy diversas formas. Circunstancia que el autor advierte en las enseñanzas mínimas: "Los contenidos mínimos que fueron formulados con esta condición carecieron de virtualidad para garantizar la formación común del alumnado, es decir, para cumplir la finalidad que la LOGSE atribuía a las enseñanzas básicas” (p. 207). Además, ante el subrayado fin curricular de asegurar la formación básica común, señala la necesidad de "una normativa básica que los desarrolle, caracterizada por la ausencia de ambigüedades y una asignación de competencias lo suficientemente clarificadora como para impedir desajustes entre la actuación de las distintas administraciones” (p. 213). El carácter mínimo de las enseñanzas parece aludir, más bien, a contenidos mínimos, en lugar de a enseñanzas que, al menos, deben ser consideradas por las distintas Administraciones educativas, a fin de garantizar la 
doble finalidad genuina del currículo. No obstante, cabe asimismo que tales enseñanzas, mínimas o comunes, se estimen a la luz de la "distinción entre lo básico imprescindible y lo básico deseable en el currículo de la educación básica” (Coll, 2006, p. 7), para subrayar, en tal caso, el carácter imprescindible de las mismas. Esa sustancia curricular, si se acepta la expresión, esa genuina entidad del currículo básico, acaso pudiera recibir la general aceptación del acuerdo.

Es necesario, además, evitar la simplificación y el prejuicio en los análisis. A modo de muestra, ni las enseñanzas comunes han de conllevar un estrecho retorno a lo básico, ni los estándares de aprendizaje deben determinar férreamente las prácticas docentes. Más cabría sostener esto último si se recuerda que el currículo de la Ley Orgánica de Ordenación General del Sistema Educativo (1990) se definía con la intención de "regular la práctica docente". Así las cosas, al carácter garantista podría unirse la naturaleza prescriptiva del currículo escolar, pero no con el objeto de determinar las prácticas docentes, sino de dar sostén legal y condición vinculante a la formación común. Y los contenidos, ya mínimos o comunes, así como los criterios de evaluación señalan o precisan su alcance.

Superar el desacuerdo y la inestabilidad en la ordenación del sistema educativo español requiere, entre otros aspectos, de mejor y mayor colaboración entre las distintas administraciones. Son necesarios por ello, acuerdos inaplazables, aunque no se alcance un consenso más amplio: convenir los elementos básicos del currículo que aseguren la formación común del alumnado; definirlos de manera expresa, sin indeterminación, generalización o ambigüedad; establecer procedimientos asimismo comunes de evaluación diagnóstica que precisen el alcance de la formación común adquirida por el alumnado; tomar decisiones y adoptar medidas, en su caso, de advertirse que no se garantiza tal formación -y su correspondiente acreditación académica- desde el desarrollo del currículo llevado a término en los procesos de enseñanza y aprendizaje. Una de las limitaciones principales del análisis realizado tiene precisamente que ver con elementos prospectivos, toda vez que el desacuerdo o la inestabilidad para la configuración del currículo, consolidados de hecho en el sistema educativo español, no auguran disposiciones proclives a corregir o superar tal estado desfavorable.

La autonomía pedagógica, sobre todo en el ámbito de los centros educativos, no puede tomar solo la forma de una declaración grandilocuente con dificultades insalvables para su ejercicio. Aparentemente, las enseñanzas mínimas, con niveles de concreción más abiertos que los de un currículo básico - cuyo diseño tiene distribuidas de manera más expresa las competencias entre las administraciones educativas y los centros-, pueden facilitar mayores grados de autonomía. Sin embargo, donde esta se verifica, y con efectos no siempre convenientes ni pertinentes, es en la formulación del currículo por las administraciones educativas, a partir de las enseñanzas mínimas, antes que en la concreción curricular en los centros. De ahí el cuestionamiento del valor referencial de las enseñanzas mínimas cuando no se articulan procesos y recursos para verificar que se garantizan en sus adecuados términos.

Asimismo, la autonomía pedagógica no debe disociarse de las condiciones para su adecuado ejercicio. Esto es, pueden formularse distintos ámbitos o aspectos a los que aplicar esa autonomía pero sin condiciones específicas para llevarla a término. De forma que se recorra un ciclo contradictorio y hasta "perverso": cesión formal de autonomía, inadecuación o insuficiencia de las condiciones de ejercicio, uso limitado o inconveniente de la autonomía, legitimación aparente para limitarla o restringirla.

Para el ejercicio de la autonomía, se reiteran condiciones relacionadas con elementos como el tiempo de dedicación y los recursos a propósito. Sin embargo, una condición sustantiva tiene que ver con la formación y cualificación profesional de quienes han de acometerla. Y, en este caso, los procesos de liderazgo, no solo relacionados con el desempeño directivo sino con el propio desarrollo profesional docente, en el marco de una extendida cultura del liderazgo pedagógico, pueden confluir en el desarrollo de la autonomía y de sus resultados.

La autonomía pedagógica no justifica disfunciones o respuestas contrarias a los principios básicos del sistema educativo o a la ordenación común del mismo. De ahí la importancia de procesos de asesoramiento y supervisión y, sobre todo, de análisis críticos de los resultados en función de las condiciones y contextos propios de los centros. Con una especial y significativa evidencia: la del valor que los centros añaden para limitar o atenuar las determinaciones o condiciones de partida. Esto es, los resultados esperados, a partir de una general consideración de tales condiciones, y los finalmente conseguidos por la singularidad de las respuestas que se sostienen en el ejercicio de la autonomía pedagógica. 
La organización del currículo del sistema educativo, que adopta, como se han analizado, distintas formas - enseñanzas mínimas, enseñanzas comunes, currículo básico-, ha de avanzar en la consideración de un conocimiento básico imprescindible que satisfaga distintos propósitos:

- La reflexión sobre la naturaleza y el alcance de los conocimientos indispensables para el desarrollo personal y social, concluida la educación obligatoria, y la continuidad formativa posterior en procesos y oportunidades de educación permanente.

- Un compromiso que garantice tanto la estabilidad como los ajustes de tal currículo, sostenido en el acuerdo.

- La autonomía pedagógica de los centros referida no solo o de manera preferente a los métodos didácticos de la enseñanza, sino asimismo al currículo más abierto y flexible que responde a las necesidades particulares de aprendizaje en contextos y situaciones diversas, con alternativas también autónomas en la organización y el funcionamiento de los centros.

Tal es, al cabo, el principal sentido de la autonomía pedagógica, aplicada a los medios que facilitan el fin último de mejorar los logros educativos del alumnado. Si bien, no cabe un maquiavelismo curricular para justificar la desnaturalización de los medios que conducen a ese fin.

\section{Referencias}

Abiétar, M. y Navas A. A. (2017). El sentido de la escolaridad obligatoria como transición o como fin. Profesorado. Revista de Currículum y Formación del Profesorado, 21(4), 75-94.

Assens, M., Carrió, V., Casal, J. D., Gasco, J., Llorente, I. y Saperas, A. (2020). Proyectos interdisciplinarios en secundaria: Una propuesta organizativa y pedagógica desde el contexto, el currículum y la autonomía. Cuadernos de Pedagogía, 505, 44-51.

Angulo, J. F. (1994). ¿A qué llamamos currículum? En J. F. Angulo y N. Blanco (Coords.), Teoría y desarrollo del currículum (pp. 17-29). Aljibe.

Apple, M. (1981). Social structure, ideology and curriculum. En M. Lawn y L. Berton (Eds.), Rethinking curriculum studies (pp. 131-159). Croom Helm.

Apple, M. (1982). Culture and economic reproduction in education. Routledge \& Kegan Paul.

Apple, M. (1986). Ideología y currículo. Akal.

Belavi, G. y Murillo, F. J. (2020). Democracia y justicia social en las escuelas: Dimensiones para pensar y mejorar la práctica educativa. REICE. Revista Iberoamericana sobre Calidad, Eficacia y Cambio en Educación, 18(3), 5-28. https://doi.org/10.15366/reice2020.18.3.001

Bloom, B. S. (1956). Taxonomy of educational objectives: Handbook 1. The cognitive domain. David McKay \& Co.

Bloom, B. S. (1964). Taxonomy of educational objectives: Volume 2. The affective domain. David McKay \& Co.

Bobbitt, F. (1918). The curriculum. Houghton Mifflin.

Bobbitt, F. (1924). How to make a curriculum. Houghton Mifflin.

Bourdieu, P. y Passeron, J. C. (1977). La reproducción. Elementos para una teoría del sistema de enseñanza. Laia.

Casanova, M. A. (2012). El diseño curricular como factor de calidad educativa. REICE. Revista Iberoamericana sobre Calidad, Eficacia y Cambio en Educación, 10(4), 6-20.

Clark, Ch. y Yinger, R. J. (1980). The hidden world of teaching: implications on teacher planning. East Lansing.

Coll, C. (1987). Psicología y currículum. Paidós.

Coll, C. (2006). Lo básico en la educación básica. Reflexiones en torno a la revisión y actualización del currículo de la educación básica. Revista Electrónica de Investigación Educativa, 8(1), 1-17.

Frías, A. S. (2007). El currículo escolar y la descentralización educativa en España. Revista de Educación 343, 199-221. 
Gagné, R. M. (1968). Educational technology as technique. En E. Eisner y E. Walance (Eds.), Conflicting conceptions of curriculum (pp. 50-63). McCutchan.

Gimeno, J. (1989). El currículum: una reflexión sobre la práctica. Morata.

Gimeno, J. (2005). La educación obligatoria: su carácter educativo y social. Morata

Gimeno, J. y Pérez Gómez, A. I. (1999). Comprendery transformar la enseñanza. Morata.

Giroux, H. (1981). Ideology, culture and the process of education. Temple University Press.

Giroux, H. (1983). Critical theory and educational practice. Deakin University.

González, T. (2004). Competencias estatales y autonómicas en la determinación del currículo o sobre las enseñanzas mínimas comunes. En VVAA., Informe educativo 2004. Análisis y situación de las Comunidades Autónomas (pp.163-195). Santillana.

Johnson, M. (1967). Definitions and models in curriculum theory. En J. R. Gress y D. E. Purpel (Eds.), Curriculum: An introduction to the field (pp. 469-485). McCutchan.

Kemmis, S. (1988). El currículum: Más allá de la teoría de la reproducción. Morata.

Martínez B. y Rodríguez, E. (2017), Los contenidos del desarrollo profesional docente: Presencias y omisiones. Profesorado. Revisa de Currículum y Formación del Profesorado, 21(3), 41-61.

Penalva, J. (2007). Análisis crítico de los aspectos teóricos del currículum flexible y abierto. Consecuencias educativas. Profesorado. Revista de Currículum y Formación del Profesorado, 11(3), 1-14.

Schön, D. A. (1983). The reflexive practitioner. How professional think in action. Temple Smith.

Schwab, J. J. (1983). Un enfoque práctico como lenguaje para el currículo. En J. Gimeno Sacristán y A. Pérez Gómez (Eds.), La enseñanza: Su teoría y su práctica (pp. 197-209). Akal.

Stenhouse, L. (1987). Investigación y desarrollo del currículo. Morata.

Taba, H. (1974). Elaboración del currículo. Troquel.

Taylor, P. H. y Richards, C. M. (1985). An introduction to curriculum studies. NFER-Nelson.

Tröhler, D. (2017). La historia del currículum como camino real a la investigación educativa internacional. Historia, perspectivas, beneficios y dificultades. Profesorado. Revisa de Currículum y Formación del Profesorado, 21(1), 202232.

Tyler, R. W. (1973), Principios básicos del currículum. Troquel.

Wheeler, D. K. (1976). El desarrollo del currículum escolar. Santillana.

Zimmer, O. (2003). A contested nation: History, memory and nationalism in Switzerland, 1798-1891. Cambridge University Press.

\section{Breve CV del autor}

\section{Antonio Montero Alcaide}

Inspector de Educación y profesor asociado en la Facultad de Ciencias de la Educación de la Universidad de Sevilla. Su tesis doctoral, sobre dirección de centros docentes, fue reconocida en los Premios Nacionales de Investigación Educativa. Esa línea de investigación se completa con el diseño y desarrollo del currículo en el sistema educativo. Es autor de libros sobre los ámbitos referidos, cuenta con artículos publicados en distintas revistas (Revista de Educación, Revista Española de Pedagogía, Revista Iberoamericana de Educación, XXI Revista de Educación, Organización y Gestión Educativa, Dirección y Liderazgo Educativo) y colabora habitualmente en publicaciones profesionales de educación (Escuela, Magisterio). Email: amontero@us.es

ORCID ID: https://orcid.org/0000-0002-3543-8139 